\title{
Sobre la presencia de Allosorus tinaei (Tod.) Christenh. (Pteridaceae, Polypodiopsida) en la provincia de A Coruña (NW ibérico)
}

\author{
Xosé Ignacio González-Martínez ${ }^{1}$ \& Carlos Boullón Agrelo ${ }^{2}$ \\ ${ }^{1}$ Avda. Miguel Rodríguez Bautista, 23. 15960, Ribeira, A Coruña, España \\ 2 r/1 da Angustia, 114. 15948, A Pobra do Caramiñal, A Coruña, España
}

\section{Correspondencia:}

X. Ignacio González-Martínez

e-mail: xoseignaciogonzalez@yahoo.es

Recibido: 22 septiembre 2018

Aceptado: 2 marzo 2020

Publicado on-line: 10 mayo 2020

Editado por:A. Enrique Salvo Tierra

\section{Resumen}

Se aporta información de interés sobre la presencia de Allosorus tinaei (Tod.) Christenh. en la provincia de A Coruña, donde constituye novedad corológica. Además, se hace una breve síntesis sobre el género Allosorus en territorio gallego. El material testigo se halla depositado en el Herbario SANT de la Universidade de Santiago de Compostela.

Palabras clave: Pteridoflora, corología, ecología, Galicia, NO ibérico.

\begin{abstract}
Presence of Allosorus tinaei (Tod.) Christenh. (Pteridaceae, Polypodiopsida) in A Coruña province (NW Iberian Peninsula)

In this note, information about Allosorus tinaei (Tod.) Christenh. in A Coruña province is provided. The discovery is a novelty for that geographical area. Furthermore, a brief summary of the genus Allosorus in Galicia is given. Collected material has been deposited at the Herbarium SANT of the University of Santiago de Compostela.
\end{abstract}

Keywords: Pteridoflora, chorology, ecology, Galicia, Iberian NW.
Como aclaración previa, en esta nota hemos seguido el actual criterio taxonómico expuesto en Eiserhardt et al. (2011), Rumsey (2014) y Gabriel y Galán et al. (2018), por el cual las distintas especies ibéricas tradicionalmente adscritas al género Cheilanthes Sw. deben ser transferidas al género Allosorus Bernh.

En la península ibérica, este grupo de helechos reúne a un colectivo de taxones termófilos de fronde pequeña y bipinnada, propios de ambientes ecológicos xéricos, con óptimo bioclimático en los pisos termo- y mesomediterráneo, y que participan, a veces como dominantes, en comunidades casmofíticas en grietas y fisuras de roquedos, tanto en sustratos ácidos como básicos (Muñoz Garmendia, 1986: 44s; Campos Saavedra \& Amigo, 2005: 66).

En lo que a Galicia respecta, las primeras referencias al género Allosorus (sub Cheilanthes) las encontramos en Merino (1909: 458s). Contribuciones posteriores (Fernández-Areces et al., 1987; Campo Saavedra \& Amigo, 2005) han ayudado a perfilar con mayor precisión el conocimiento sobre su distribución y sinecología en nuestra región, donde hasta la fecha se conocen un total de 4 especies: $A$. acrosticus (Balb.) Christenh., presente en los asomos calizos de la provincia de Ourense, A. guanchicus (Bolle) Christenh, con una pequeña población aislada en el término municipal de Sober, al sur de la provincia de Lugo, A. hispanicus (Mett.) Christenh. y A. tinaei (Tod.) Christenh., estas dos últimas, si bien escasas, más ampliamente repartidas (Muñoz Garmendia, 1986: 45s; Fernández-Areces et al., 1987: 212; Amigo, 2005: 13s; Camaño et al., 2005: 44; Pino Pérez et al., 2007: 63; Romero, 2008: 120; Campo Saavedra \& Amigo, 2009: 72; Gómez Vigide, 2016: 314; Anthos [2020]). Sobre la presencia de $A$. guanchicus en los afloramientos calizos de Rubiá (Ourense) (Fernández Areces et al., 1985: 216, Tb. $3)$, parece que en realidad los materiales colectados por estos autores deben ser interpretados como el netamente calcícola $A$. acrosticus (Campos Saavedra \& Amigo, 2005: 69), bien conocido de ese territorio ubicado en el subsector Berciano (Giménez de Azcárate \& Amigo, 1996: 48s). Lo mismo sucede con A. pteridioides (Reichard) Christenh. $(=\mathrm{Ch}$. maderensis Lowe), que ha sido señalado en varias ocasiones para el oriente gallego. Así, Laínz (1971: 38) lo indica para Quiroga (Lugo) y Sáenz de Rivas \& Rivas-Martínez (1979: 227) y Fernández Areces et al. (1987: 216, Tb. 3) lo citan en las calizas del municipio de Rubiá (Ourense). Por otro lado, su presencia en estas localidades gallegas también aparece recogida, en forma de cuadrículas UTM $10 \times 10 \mathrm{~km}$, en el Atlas de pteridófitos elaborado recientemente por Moreno Saiz et al. (2015: 26, Fig. 2, Mapa 30, sub Ch. 
maderensis Lowe). Sin embargo, parece que finalmente $A$. pteridioides no es especie gallega, al menos por el momento (Campo Saavedra \& Amigo, 2009: 69), y de nuevo, para el caso de las citas en los asomos calizos orensanos, habría que hablar en realidad de $A$. acrosticus. En este sentido, y reforzando lo antedicho, Flora iberica (Muñoz Garmendia, 1986: 47) no señala a esta especie para el territorio de Galicia.

En relación al tratamiento de la especie aquí reseñada, se aportan las coordenadas UTM del lugar de recolección [X, Y (m), 29T; ETRS89], la altitud (m s. n. m.), ecología, especies acompañantes, fecha de recolección, colectores y número identificador de herbario. Para la adscripción fitosociológica de la comunidad en la que hemos encontrado a este helecho, se han seguido las propuestas sintaxonómicas de Fernández Areces et al. (1987) e Izco et al. (1999). Con fines corológicos, se han consultado los portales electrónicos de datos Anthos (Sistema de información de las plantas de España, www.anthos.es) y GBIF (Global Biodiversity Information Facility, www.gbif.org). El material testigo se halla depositado en el Herbario SANT de la Universidade de Santiago de Compostela.

El objetivo principal de esta nota es actualizar la distribución de $A$. tinaei (Figura 1) en el ámbito de Galicia.

\section{Allosorus tinaei (Tod.) Christenh}

ESPAÑA. A Coruña. Santiso, Monte da Pena, en las proximidades del río Ulla, cerca del embalse de Portodemouros, en el límite con la provincia de Pontevedra, 576657, 4743882, 290 m, comunidad casmofítica, probablemente adscribible a la alianza Cheilanthion hispanicae, instalada en grietas de rocas ultrabásicas, en compañía de otras especies rupícolas como Sedum hirsutum, Umbilicus rupestris, los musgos Racomitrium cf. lanuginosum y Polytrichum aff. piliferum, y el liquen Cladonia gr. pyxidata, 13-III-2018, Carlos Boullón Agrelo \& X. Ignacio González-Martínez (SANT 75808).

Hasta el momento, y al igual que el resto de congéneres gallegos, la presencia de $A$. tinaei se hallaba circunscrita al sistema Miño-Sil (Fernández Areces et al., 1987: 212; Morales \& Fernández Casas, 1989: 69s; Amigo et al., 2004: 193; Moreno Saiz et al., 2015: 27; Anthos [2020]; GBIF [2020]), por lo que el presente testimonio, ya fuera de dicha cuenca hidrográfica, supone novedad corológica para la provincia de A Coruña, ampliándose de forma sensible su reducida área de distribución en nuestro territorio. A día de hoy puede decirse que en Galicia se halla presente en un total de 16 cuadrículas UTM de $10 \times 10$ km (Amigo, 2005: 16; dat. prop.).

En Galicia, el área de distribución de $A$. tinaei suele solaparse con la de $A$. hispanicus, una especie morfológicamente muy parecida a la primera y con la que a menudo se confunde. Sin embargo, $A$. tinaei se caracteriza por presentar la cara abaxial de la lámina de la fronde provista de pequeños pelos glandulares distribuidos de forma dispersa, mientras que $A$. hispanicus tiene pelos largos que prácticamente tapizan dicha cara de la fronde (Sáenz de Rivas \& Rivas-Martínez, 1979: 223; Muñoz Garmendia, 1986.: 45s; Amigo et al., 2004: 193; Prelli, 2015: 100s).

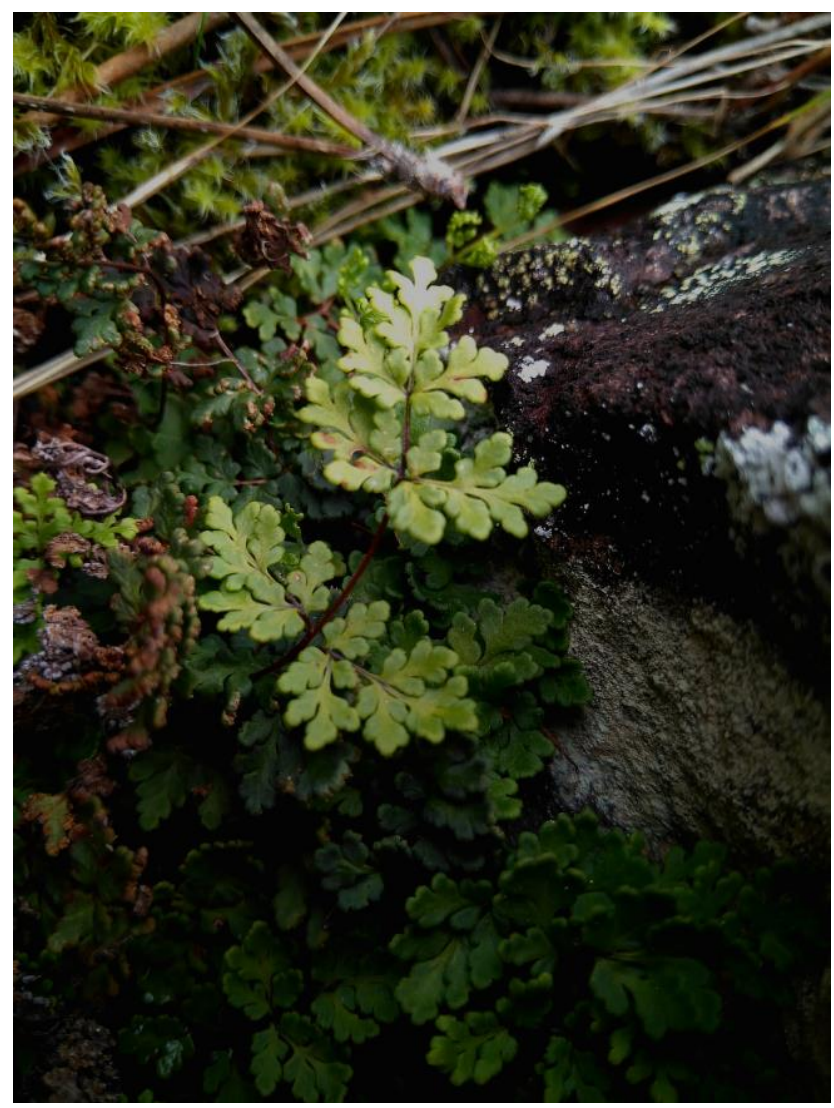

Figura 1. Allosorus tinaei (Tod.) Christenh., grietas de rocas serpentínicas (Santiso, Melide, A Coruña; 13-III-2018).

Figure 1. Allosorus tinaei (Tod.) Christenh. Ultrabasic rocks crevices (Santiso, Melide, A Coruña; 13-III-2018).

En el lugar de recolección lo encontramos de forma escasa ocupando grietas de rocas ultrabásicas (sustratos serpentínicos), en la ladera de solana del valle del río Ulla, en un escenario ecológico de marcada mediterraneidad bioclimática, con presencia de especies termófilas como Arbutus unedo L. y Quercus suber L. Por otra parte, la posible presencia de este helecho en el valle fluvial del río Ulla ya había sido vaticinada por Amigo et al. (2004: 195). Todo parece indicar que la localidad más próxima a la nuestra se encuentra aproximadamente a 33,5 km en línea recta, cerca del lugar de Xillán, en la parroquia lucense de Pedrafita, municipio de Chantada (UTM: 603228,4723617, c. $350 \mathrm{~m}$ ) (dat. prop.).

Finalmente, podría resultar interesante intensificar la búsqueda de este u otros Allosorus en toda esta área de la Galicia Central, rica en afloramientos serpentínicos y conocida además por albergar numerosos taxones amenazados y/o de elevadísimo valor biogeográfico. 


\section{Agradecimientos}

Al personal del Herbario SANT de la Universidade de Santiago de Compostela, por su amabilidad, diligencia y siempre caluroso acogimiento. Así mismo, agradecer el tratamiento editorial, y personal, recibido por el Profesor Enrique Salvo Tierra, siempre atento a ayudarnos con nuestras dudas. Finalmente, a los dos revisores anónimos cuyas sugerencias han ayudado de forma sustancial a la mejora del manuscrito de partida.

\section{Bibliografía}

Amigo, J., Quintanilla, L.G. \& Romero-Buján, M.I. (2004). Pteridófitos (División Pteridophyta). In A. Rigueiro (Ed.), Proyecto Galicia, 42. Botánica II: 165-255. A Coruña, Galicia: Hércules Ediciones S.A.

Amigo, J. (2005). Los helechos en el noroeste ibérico y su interés para la conservación. Recursos Rurais (IBADER), 2, 11-22.

Anthos. [2020]. Sistema de información de las plantas de España. Real Jardín Botánico, C.S.I.C.Fundación Biodiversidad. Recurso electrónico en www.anthos.es. Consulta realizada en [20-II2020].

Camaño, J.L., Pino Pérez, J.J., Silva-Pan F.J. \& Pino Pérez, R. (2005). Asientos corológicos. LOU 2003. Boletín BIGA, 1, 2-138.

Campo Saavedra, P. \& Amigo, J. (2009). Sobre el aislamiento de la población de Cheilanthes guanchica C. Bolle (Sinopteridaceae, Monilophyta) en Galicia. Nova Acta Científica Compostelana (Bioloxía), 18, 65-73.

Eiserhardt, W.L., Rohwer, J.G., Russell, S.J., Yesilyurt, J.C. \& Schneider, H. (2011). Evidence for radiations of cheilanthoid ferns in the Greater Cape Floristic Region. Taxon, 60, 1269-1283.

Fernández-Areces, P., Pérez-Carro, F.J. \& DíazGonzález, T.E. (1987). Estudio del Cheilanthion hispanicae Rivas Goday 1955 em. Sáenz de Rivas \& Rivas-Martínez 1979 y comunidades afines, en el Sector Orensano-Sanabriense (Provincia Carpetano-Ibérico-Leonesa). Lazaroa, 7, 207-220.

Gabriel y Galán, J.M., Molino, S., de la Fuente, P. \& Seral, A. (2018). Novedades para la pteridoflora ibérica en el contexto de un nuevo sistema para las plantas vasculares sin semilla. Botanica Complutensis, 42, 69-81.
GBIF [2020]. The Global Biodiversity Information Facility. Recurso electrónico en www.gbif.org. Consulta realizada en [23-II-2020].

Giménez de Azcárate J. \& Amigo, J. (1996). Inventario da flora vascular dos afloramentos calíos de Galicia (Pteridophyta e Spermatophyta). Cadernos da Área de Ciencias Biolóxicas, XII, 1181.

Gómez Vigide, F. (2016). El Herbario FGV. Boletín $B I G A, 15,1-336$.

Laínz, M. (1971). Aportaciones al conocimiento de la flora gallega, VII. Anales del Instituto Forestal de Investigaciones y Experiencias, 12, I-39. Madrid.

Merino, B. (1909). Flora descriptiva e ilustrada de Galicia. Vol. III. Santiago de Compostela, Galicia: Tipografía Galaica.

Morales, M.J. \& Fernández Casas, J. (1989). Asientos para un Atlas Corológico de la Flora Occidental. Mapa 291. Fontqueria, 25, 68-72.

Moreno Saiz, J.C., Pataro, L. \& Pajarón Sotomayor, S. (2015). Atlas de de los pteridófitos de la Península Ibérica e Islas Baleares. Acta Botanica Malacitana, 40, 5-55.

Muñoz Garmendia, F. (1986). Cheilanthes Sw. In S. Castroviejo, M. Laínz, G. López González, P. Montserrat, F. Muñoz Garmendia, J. Paiva \& L. Villar (Eds.), Flora iberica I. LycopodiaceaePapaveraceae (pp. 44-51). Real Jardín Botánico, Madrid: C.S.I.C.

Pérez-Carro, F.J., Fernández Areces, P., Díaz, T.E. \& Salvo, A.E. (1985). Aportación al conocimiento del género Cheilanthes en la Península Ibérica. Acta Botanica Malacitana, 10, 27-32.

Pino Pérez, J.J., Camaño, J.L. \& Pino Pérez, R. (2007). Asientos corológicos, LOU 2004. Boletín $B I G A, 2,35-109$.

Prelli, R. (2015). Guide des Fougères et plantes alliées. France, FRA: Belin.

Romero, M.I. (2008). Catálogo da Flora de Galicia. Monografías do IBADER, 1. Universidade de Santiago de Compostela, Lugo, Galicia: IBADER.

Rumsey, F. (2014). Some name changes in the Pteridaceae of Macaronesia and Europe - with validation of hybrid names in the genus Allosorus Bernh. Fern Gazette, 19, 275-279.

Sáenz de Rivas, C. \& Rivas-Martínez, S. (1979). Revisión del género Cheilanthes (Sinopteridaceae) en España. Lagascalia, 8, 215241. 